\title{
Addition to the Special Issue on the CTP network consisting of reviews and original papers emerging from the CTPIOD meeting (Contribution To Progress in Obesity and Diabetes Research) 2016
}

The following article is a contribution to the Special Issue on the CTP network consisting of reviews and original papers emerging from the CTPIOD meeting (Contribution To Progress in Obesity and Diabetes Research) 2016 but was erroneously published in

Journal of Physiology and Biochemistry

December 2016, Volume 72, Issue 4, pp. 803-812

DOI 10.1007/s13105-016-0518-0

Increased urinary lysophosphatidic acid in mouse with subtotal nephrectomy: potential involvement in chronic kidney disease

Koryun Mirzoyan · Anna Baïotto · Aude Dupuy · Dimitri Marsal · Colette Denis · Claire Vinel · Pierre Sicard · Justine Bertrand-Michel · Jean-Loup Bascands · Joost P. Schanstra · Julie Klein · Jean-Sébastien Saulnier-Blache

Received: 21 June 2016 /Accepted: 9 September 2016 /Published online: 17 September 2016 